\title{
Anabases
}

ANABASES Traditions et réceptions de l'Antiquité

18 | 2013

Varia

\section{Les gypsothèques universitaires, diffusion d'une Antiquité modèle}

Soline Morinière

\section{OpenEdition}

1 Journals

Édition électronique

URL : http://journals.openedition.org/anabases/4360

DOI : 10.4000/anabases.4360

ISSN : 2256-9421

Éditeur

E.R.A.S.M.E.

Édition imprimée

Date de publication : 1 octobre 2013

Pagination : 71-84

ISSN : 1774-4296

\section{Référence électronique}

Soline Morinière, "Les gypsothèques universitaires, diffusion d'une Antiquité modèle ", Anabases [En ligne], 18| 2013, mis en ligne le 01 novembre 2016, consulté le 21 octobre 2019. URL : http:// journals.openedition.org/anabases/4360 ; DOI : 10.4000/anabases.4360

(c) Anabases 
Anabases 18 (2013), p. 71-84

\section{Les gypsothèques universitaires, diffusion d'une Antiquité modèle}

SOLINE MORINIÈRE

«Le Muséum n'est point un vain rassemblement d'objets de luxe et de frivolité qui ne doivent servir qu'à satisfaire la curiosité. Il faut qu'il devienne une école importante. Les instituteurs y conduiront leurs jeunes élèves, le père y mènera son fils ${ }^{1}$. "

JACQUeS-Louis DAVID, membre de la commission du Muséum central des arts exprimait dès 1793 en ces mots l'objectif pédagogique auquel devait tendre le musée. Conservatoires du patrimoine, lieux de formation libre et indépendante des artistes, les musées ont aussi et surtout vocation à éduquer le goût des citoyens. Le Dictionnaire de l'Académie française définissait en 1823 le musée comme "un lieu destiné soit à l'étude des lettres, des sciences et des beaux-arts, soit à rassembler les productions, les monuments qui y sont relatifs ». Le XIX ${ }^{\mathrm{e}}$ siècle, âge d'or des musées, voit fleurir ce type d'établissements dans toutes les grandes villes, et même dans les lieux d'enseignement supérieur. Des musées d'anatomie sont créés dans les facultés de médecine; des séries minéralogiques, géologiques et les instruments nécessaires aux recherches en physique et en chimie composent les collections des facultés de sciences; tandis que celles de Lettres rassemblent des cartes, globes et atlas pour la géographie, des moulages, objets originaux et photographies pour l'archéologie et l'histoire de l'art. Ne semble-t-il pas évident que ces musées, créés en vue d'un enseignement, devaient jouer leur rôle non

1 J.-L. DAvid, Convention nationale. Second rapport sur la nécessité de la suppression de la commission du Muséum dans la séance du 27 nivôse, l'an II de la république française, Paris, Impr. nationale, 1793. p. 4. 
seulement en faveur des étudiants, mais aussi d'un public extérieur, artistes et amateurs, population locale et visiteurs étrangers ? C’est la réflexion qui a présidé à la création de ces collections si particulières.

De tous ces musées universitaires, nous ne retiendrons pour la présente étude qu'un seul type : les collections de moulages des facultés de lettres, c'est-à-dire la réunion, dans un même espace, de reproductions en plâtre, en gypse - ce qui leur a donné le nom de gypsothèques - de sculptures de différentes périodes artistiques dispersées en divers lieux. Fondées dans la seconde moitié du siècle, suite à l'introduction de l'archéologie et de l'histoire de l'art comme discipline universitaire, elles ont comme domaine de prédilection les arts grec et romain et sont alors, auprès des étudiants, des artistes et du grand public, un vecteur de diffusion d'une Antiquité classée, organisée et réfléchie par les professeurs, en quelque sorte une "Antiquité modèle ". À travers ces collections, c'est la conception et la définition même de l'Antiquité que l'on peut appréhender, sa place et son importance dans les lieux d'enseignement supérieur et les modalités de sa transmission et de sa réception auprès des différents publics.

\section{L'Antiquité au cœur de l'enseignement universitaire}

L'archéologie apparaît officiellement dans les Universités en 1876 avec la création de quatre cours complémentaires, à Paris tout d'abord (décret du 6 mars 1876), suivie par Bordeaux et Lyon (31 octobre 1876), puis Toulouse ( $1^{\text {er }}$ novembre 1876). Elle intègrera avant 1900 , parfois difficilement, tous les autres centres universitaires ${ }^{2}$. Cet enseignement s'identifie d'abord comme une science auxiliaire de l'histoire ${ }^{3}$, qu'elle aide à comprendre par l'étude des monuments figurés, avant de trouver sa propre autonomie et de se scinder progressivement - non sans ambiguïté - en deux : l'archéologie et l'histoire de l'art ${ }^{4}$, tout en élargissant son champ d'étude aux périodes artistiques postérieures.

2 Sur les résistances des universités face à l'introduction de l'archéologie, voir E. PERrinSAMINADAYAR, "Les résistances des institutions scientifiques et universitaires à l'émergence de l'archéologie comme science ", in Rêver l'archéologie au XIX siècle : de la science à l'imaginaire, Saint-Étienne, université de Saint-Étienne, 2001, p. 47-64.

3 Dès l'origine, malgré son introduction au milieu des disciplines littéraires, l'archéologie est perçue comme une science qui demande rigueur et méthode. Voir È. Gran-Aymerich, Naissance de l'archéologie moderne, 1798-1945, Paris, éd. du CNRS, 1998.

4 L. ThERrien, L'histoire de l'art en France : genèse d'une discipline universitaire, Paris, CTHS, 1998. Cette confusion entre l'archéologie et l'histoire de l'art est visible dans les annuaires et livrets des universités, qui donnent l'intitulé des cours. Albert Dumont, dans son allocution à l'ouverture du cours d'archéologie à l'École de Rome en 1873, exprime la différence fondamentale entre les deux disciplines comme une divergence de regard et de méthode : l'histoire de l'art porte sur les œuvres empreintes du "sentiment du beau " et se concentre sur ses caractères généraux et absolus, tandis que l'archéologie considère 
Par l'étude des sujets de cours donnés à l'époque, répertoriés dans les annuaires et livrets des universités, la prédominance quasi exclusive de l'Antiquité grecque est évidente. Dans l'enseignement général des facultés de lettres, nombreux sont les cours liés aux civilisations grecque et romaine (institutions, épigraphie, littérature classique, etc.). L'art de la Grèce, largement diffusé dans le goût public depuis la Renaissance, comme l'ont montré Nicholas Penny et Francis Haskell dans leur étude de référence ${ }^{5}$, est porté aux nues par Johann Joachim Winckelmann (1717-1768) dans son ouvrage Geschichte der Kunst des Altertums ${ }^{6}$. Les théories de cet historien de l'art allemand ont longtemps régi la vision de l'art antique et se ressentent dans l'enseignement universitaire : la part belle est à l'art grec, considéré par Winckelmann comme supérieur aux productions artistiques des autres peuples. La classification opérée par le théoricien d'un art grec en trois phases est également suivie, et la période classique des $\mathrm{V}^{\mathrm{e}}$ et $\mathrm{IV}^{\mathrm{e}}$ siècles, perçue comme l'apogée de cette civilisation, est la plus fréquemment étudiée.

Le XIX ${ }^{\mathrm{e}}$ siècle, riche en découvertes archéologiques, est l'occasion pour ces professeurs - qui ne sont autres que des anciens élèves de l'École française d'Athènes et des archéologues de terrain - de renouveler la vision de l'Antiquité et de bouleverser les certitudes antérieures. De ce fait, de nombreux cours portent sur l'actualité des fouilles archéologiques réalisées par les grandes nations européennes à Délos, à Delphes, à Athènes, à Olympie, en Asie Mineure, etc. Les autres civilisations abordées - égyptienne, orientale et romaine - ne s'expliquent alors que par rapport à la Grèce, les deux premières considérées comme les origines de l'art hellénique, la dernière comme son prolongement.

Des collections dites " archéologiques " sont réunies par les professeurs. Elles seront dans un premier temps, et toujours majoritairement, consacrées à l'art antique même si les cours portent sur des sujets plus étendus. Le manque de moyens financiers et de place sont les principales raisons de cette restriction. Il semble alors plus avantageux, au lieu de réunir quelques œuvres éparses de toutes époques de l'art, de constituer une collection complète sur une seule période artistique : l'art antique, c'est-à-dire le sujet prédominant des cours et l'art le moins représenté dans les musées et monuments parisiens et provinciaux ${ }^{7}$.

l'ensemble de la production humaine et se penche davantage sur ses caractères particuliers et relatifs.

5 Fr. Haskell et N. Penny, Taste and the Antique : the lure of classical sculpture 1500-1900, New Haven ; London, Yale university press, 1981.

6 J. J. Winckelmann, Geschichte der Kunst des Altertums, Dresden, Walther, 1764.

7 H. LECHAT, La sculpture grecque : à propos des musées de moulages des universités françaises. Leçon d'ouverture du cours d'Histoire de l'art professé à la faculté des lettres, Lyon, impr. A. Storck \& Cie, 1898, p. 1-2. 


\section{Les collections de moulages, supports de l'enseignement}

Ces collections ont des rôles multiples, dont l'illustration des cours et l'application de la méthode scientifique ne sont pas les moindres. Le principe premier est simple : c'est par la vue que l'élève assimile au mieux les connaissances. Cette réflexion n'est pas seulement propre à la discipline archéologique ; dans tous les domaines, cet enseignement par l'image est réputé meilleur que de longues dissertations et d'importants opus textuels. Il est largement répandu par un ministre de l'Instruction publique, Victor Duruy (1811-1894), qui favorise d'une part la publication d'ouvrages illustrés et d'autre part la création de laboratoires de recherche dans les universités pour " placer à côté de l'enseignement théorique les exercices qui peuvent le fortifier et l'étendre ${ }^{8}$ ". Pour l'archéologie, c'est une évidence ; l'étudiant intègre, par l'étude visuelle et la pratique, les compétences inhérentes à tout archéologue et historien de l'art : "Par cette éducation des yeux il acquiert cette finesse de tact, cette sûreté de goût et de jugement, cette connaissance précise que toute l'érudition du monde est incapable de donner ${ }^{9}$."

À sa suite, Albert Dumont (1853-1929), alors directeur de l'École française d'Athènes, affirme qu' "il n'y a pas d'enseignement de l'archéologie sans collection de moulages. [...] L'histoire de l'art s'apprend par les yeux plus encore que par les leçons du maître; il faut que l'élève s'habitue à voir les différences des styles, la succession des formes ${ }^{10}$ ". Sa pensée est largement partagée par tous les élèves de l'École française d'Athènes, qui deviennent professeurs d'archéologie dans les universités, notamment Maxime Collignon (1849-1917), chargé de cours à la faculté des lettres de Bordeaux puis professeur à la Sorbonne, initiateur de la collection de moulages de l'université bordelaise et fondateur du musée d'art antique de la seconde, qui défend activement ce principe d'affilier à l'enseignement théorique des collections de moulages, supports de " démonstrations ${ }^{11}$ ". L'archéologie est en effet une science d'observation, pour laquelle deux méthodes sont applicables : la première, dite artistique ou "critique des copies ",

8 J. AdHÉmar, "L'enseignement par l'image ", in Gazette des Beaux-Arts, septembre 1981. p. 49-60. Victor Duruy, ministre de l'Instruction publique de 1863 à 1869 , a beaucoup œuvré pour la gratuité des écoles primaires et l'éducation des filles. Il commença également à réformer l'enseignement supérieur, augmentant les moyens financiers et matériels des universités et créant l'École pratique des Hautes Études. Pour une biographie de Victor Duruy, voir J.-C. GESLot, Victor Duruy Historien et ministre (1811-1894), Villeneuve d'Asq, Presses universitaires du Septentrion, 2009.

9 Ch. Dient, "Cours d'archéologie. Leçon d'ouverture ", in Annales de l'Est, $2^{\mathrm{e}}$ année, Nancy, Berger-Levrault et Cie, 1888. p. 42.

10 A. Dumont, "Les moulages du musée du Louvre ", in La Gazette des Beaux-arts, Paris, 1875, p. 419-420.

11 Sa mission effectuée en 1882 pour le compte du ministère de l'Instruction publique n'est pas étrangère à cette conviction. M. ColLIGNON, "L'enseignement de l'archéologie classique et les collections de moulages dans les universités allemandes ", in Revue internationale de l'enseignement, Paris, Société de l'enseignement supérieur, 1882, vol. 2. p. 256-270. 
s'appuie sur un classement des œuvres par analogies pour en étudier par comparaison les caractères communs et originaux et déterminer par là-même les transformations d'un même type artistique, le style d'un sculpteur, restituer son corpus, dater une sculpture par chronologie relative et replacer l'œuvre dans son contexte et dans le développement de l'art grec ${ }^{12}$; la seconde est historique et s'attache à étudier les découvertes archéologiques d'un même site, pour déterminer les caractéristiques d'un style ou d'une école et rendre compte des mœurs, des croyances et des usages d'une société et d'une époque $^{13}$. La tridimensionnalité des moulages permet de se faire une idée plus fidèle du volume et des contours de l'original, et la blancheur des plâtres de faire abstraction des différents matériaux des œuvres reproduites, notamment pour les copies romaines en marbre d'originaux grecs initialement en bronze. En ce sens, le plâtre permet de mettre toutes les œuvres au même niveau, et il se substitue à l'original. L'enseignement de l'archéologie est divisé généralement en trois cours : deux sur des questions générales (l'un public, l'autre réservé aux seuls étudiants) et une séance d'exercices pratiques dans le musée de moulages. Le professeur fait cours devant les œuvres, les décrivant et les analysant de visu, ou demandant aux élèves de faire de même. C'est aussi sur les moulages qu'ils ont à disposition dans leurs laboratoires de recherches que se penchent ces savants pour leurs études personnelles et leurs essais de reconstitutions.

La sélection des œuvres est alors stratégiquement réfléchie. Aucun professeur n'a pour velléité de réunir toutes les sculptures archéologiques découvertes depuis des siècles et d'en faire un musée universel. Leur objectif est de constituer un outil qui leur serve tant pour leur cours que pour leur recherches personnelles. Ils sélectionnent des exemples choisis des arts utiles à leur enseignement, non seulement des poncifs de la statuaire grecque (la Victoire de Samothrace, la Vénus de Milo, l'Apollon du Belvédère, les sculptures du Parthénon), mais aussi des œuvres récemment découvertes, illustrant l'actualité archéologique (Corai et Couroï de l'Acropole d'Athènes, sculptures des fouilles françaises à Delphes et allemandes à Pergame et Olympie, etc.) ${ }^{14}$. La vue de salle du musée d'art antique de l'université de Paris (fig. 1) montre ainsi un des reliefs les plus célèbres de la frise de la Gigantomachie de l'Autel de Pergame et plusieurs figures des frontons du Temple de Zeus à Olympie. Découvertes récentes universellement reconnues, elles sont présentes dans la plupart des collections universitaires françaises.

12 "Allocution prononcée à l'ouverture du cours d'archéologie à l'École de Rome en 1873 ", in A. Dumont, Notes et discours 1873-1884, Paris, Armand Colin et C $C^{\mathrm{ie}}, 1885$.

13 Ch. DieHL, 1988, op. cit., p. 29-30.

14 "Le plus riche musée du monde n'offre que des exemples trop particuliers. Réunissez au contraire pour chaque époque les types les plus clairs, les œuvres les plus parfaites ; classezles selon l'ordre du temps, il suffit d'un catalogue de quelques pages pour vous apprendre avec sûreté l'histoire de l'art ». A. DumOnt, 1875, op. cit., p. 420. 


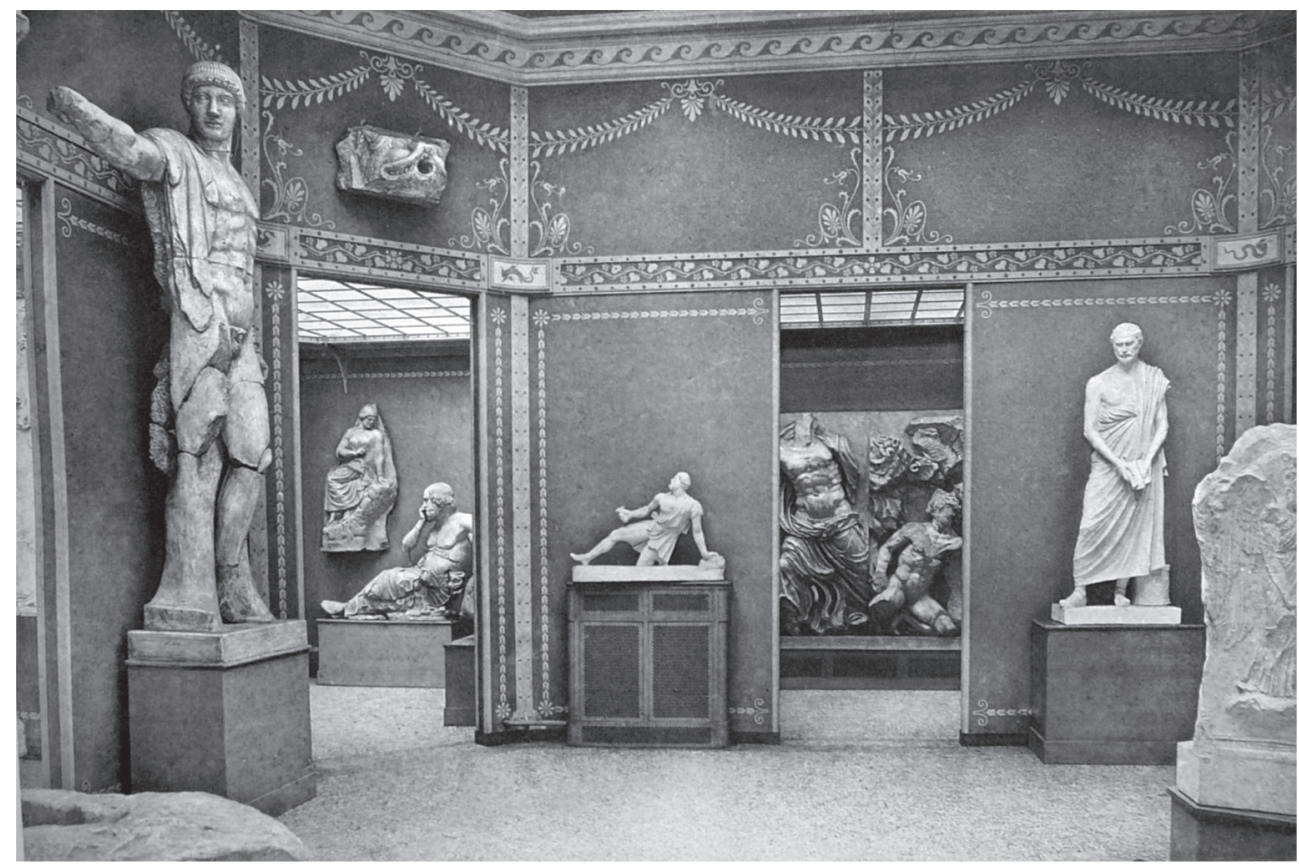

Figure 1. Le musée d'art antique de la faculté des lettres de Paris (C) H.-P. Nenot, Monographie de la Nouvelle Sorbonne, Paris, Impr. nationale, 1903, pl. XXXVII

\section{Musées d'art, lieux d'éveil pour les artistes}

L'intérêt des gypsothèques universitaires ne saurait se réduire aux seuls étudiants des facultés. Véritables temples des arts et de la Beauté, les collections de moulages d'antiques sont pour les artistes et élèves des Écoles de Beaux-arts une source d'inspiration, qui leur offre des modèles pour l'un des deux exercices académiques traditionnels, l'étude de la figure humaine d'après l'antique, plus communément appelé le dessin d'après la bosse. Pour cette épreuve, les professeurs étaient particulièrement attachés à quelques chefs-d'œuvre de la statuaire grecque, présents dans tous les musées de moulages universitaires mais aussi dans les propres collections des Écoles de Beaux$\operatorname{arts}^{15}$. Bien que l'on sache qu'elle existe ${ }^{16}$, il est difficile de rendre compte aujourd'hui

15 Elles sont attestées à Bordeaux, Montpellier, Toulouse, Lyon et Paris dans les Écoles de Beaux-arts, à Lille dans l'École régionale des arts plastiques.

16 François Benoît évoque les cours de dessin et les ateliers de modelage qui se faisaient dans l'Institut d'histoire de l'art de l'université de Lille. F. BENOîT "L'enseignement de l'histoire de l'art et l'Institut d'histoire de l'art de l'université de Lille ", in Revue internationale de l'enseignement, vol. 41, Paris, Société de l'Enseignement supérieur, 1901, p. 538. 
de la fréquentation des gypsothèques universitaires par les élèves des Beaux-arts. On la dit assidue pour quelques-unes d'entre elles, mais les documents qui l'énoncent sont rédigés par les universitaires et nous approchons de trop près le risque d'une exagération consciente, permettant de redorer le prestige d'une Université au sein du climat de concurrence de l'époque. Le cas de l'université de Bordeaux est emblématique : elle affirme que les élèves des Beaux-arts viennent nombreux visiter son musée archéologique mais déplore par ailleurs l'exiguïté des locaux affectés aux collections. Ce manque de place, visible sur les photographies anciennes (fig. 2), n'est pas adapté à la pratique du dessin car il suppose une absence de recul ${ }^{17}$. En réalité, il eût fallu que l'Université eût davantage à offrir au regard de ces jeunes artistes pour qu'ils prissent la peine de s'y déplacer. La volonté d'une complémentarité des collections est parfois évoquée ${ }^{18}$, ou même à terme une fusion des deux collections qui ne fut cependant jamais réalisée $^{19}$, les détracteurs arguant que les principes de présentation entre les Beaux-arts et la science archéologique sont trop divergents ${ }^{20}$.

17 H. De La Ville de Mirmont et P. Paris « Le musée de moulages de Bordeaux », in Revue internationale de l'enseignement, Paris, Société de l'Enseignement supérieur, 1899, vol. 38, p. $513-515$.

18 À Montpellier, le doyen Ferdinand Castets explique que le musée universitaire se focalisant sur la période antique, l'École régionale des Beaux-arts pourrait développer davantage ses acquisitions en faveur d'œuvres de la Renaissance. S. Moriniere, Le Musée des Moulages de la faculté des lettres de Montpellier : origine et développement d'une collection universitaire (1890-1904). Mémoire d'étude, École du Louvre, 2010, vol. 1, p. 27-28.

19 Projet évoqué à Bordeaux et favorisé par la nomination du professeur d'archéologie Pierre Paris à la direction de l'École municipale des Beaux-arts et des Arts décoratifs. L'affaire est portée devant le Conseil de l'Université en 1898. Cependant, malgré l'agrément du Conseil et de l'administration municipale des Beaux-arts, le projet ne se concrétisa pas. H. De La Ville de Mirmont et P. Paris, 1899, op. cit., p. 514.

20 À Paris, chaque musée de moulage (musée du Louvre, École des Beaux-arts, Université) clame son caractère spécifique pour légitimer son existence. Ainsi, bien que les étudiants aient accès à la salle des moulages du Louvre et au musée des études de l'École des Beauxarts, ces collection n'ayant "pas été faites en vue de l'enseignement supérieur ; elles sont loin de répondre à ses besoins » déclarait Louis Liard pour justifier la création du musée universitaire. L. LIARD, "Les bienfaiteurs de l'université de Paris ", in La Revue de Paris, $20^{\mathrm{e}}$ année, tome 2, Paris, mars-avril 1913, p. 346. Et le Sous-secrétaire d'État aux BeauxArts, Étienne Dujardin-Beaumetz déclarait que l'objectif du musée des plâtres du Louvre était " très différent de celui de l'École des Beaux-arts, où il s'agit d'un enseignement spécial, non pas du grand public des musées nationaux ; et tout autre que le but archéologique, scientifique de la Sorbonne ». Ch. Georgel, "Le musée, lieu d'enseignement, d'instruction et d'édification ", in Ch. GEORGEL (dir.), La jeunesse des musées : Les musées de France au XIX siècle, Paris, Éditions de la réunion des musées nationaux, 1994, p. 58-70. 


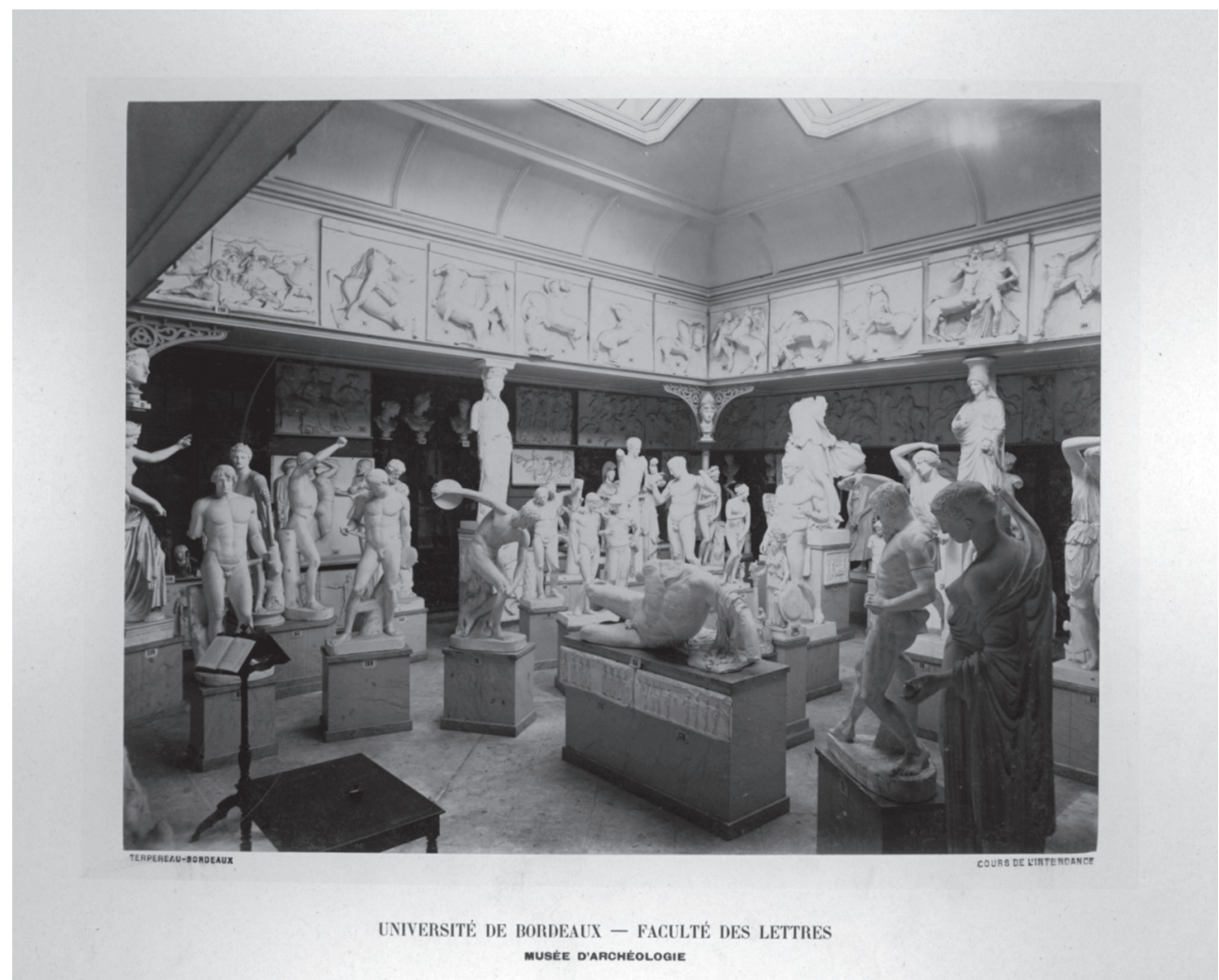

Figure 2. TERPEREAU Jules-Alphonse.

Musée archéologique de la faculté des lettres de Bordeaux. ca 1886.

(C) Université Bordeaux 3

Cependant, ces collections sont bien plus qu'un simple répertoire de modèles, elles deviennent un moyen de s'immerger dans la période de l'art considérée depuis la Renaissance comme le modèle de l'idéal absolu. "Seuls les musées de moulages peuvent donner à l'artiste le sentiment de ce qu'est l'art grec par opposition à l'art romain. [...] Pour se pénétrer de la beauté grecque, il ne suffit pas de regarder un torse, un faune, une stèle funèbre, il faut avoir sous les yeux une longue suite d'œuvres vraiment grecques ${ }^{21}$. " Il y a là peut-être la spécificité des collections universitaires par rapport à celles des Beaux-arts, leur caractère plus complet et leur organisation scientifique

21 A. Dumont, 1875, op. cit., p. 420. Fort de la même conviction, Jules Ferry reprendra cet argument dans un rapport sur la création d'une caisse des musées en 1890 . Il y a là la raison de la présence de moulages dans les musées de Beaux-arts, à Nemours, Chaumont, Angers pour ne citer que trois exemples. Ils servaient à combler les lacunes. Ch. GeORGEL, op. cit., in Ch. GeOrgel (dir.), op. cit., 1994, p. 58-70. 
qui transmettent par la vue l'évolution de la sculpture antique. Cette destination des collections de moulages universitaires pour l'usage des artistes perdure pendant toute la première moitié du Xx $\mathrm{x}^{\mathrm{e}}$ siècle ; dans les années 1950 on organisait encore des cours de dessin au milieu des moulages du musée universitaire lyonnais.

\section{Des collections publiques, lieux de vulgarisation artistique et de "moralisation"}

Dès les origines de ces collections, les professeurs des facultés ont pour objectif une ouverture auprès d'un public plus large, en vue d'une éducation esthétique et artistique de la population. Certes, la raison était également financière car si elles répondaient aux besoins de l'enseignement, et aux nécessités de la recherche, les ouvrir au grand public était un moyen d'appuyer les demandes de subventions municipales, régionales et étatiques en faisant de ce projet une œuvre d'intérêt général ${ }^{22}$. Mais elle s'intègre aussi dans des schémas de pensée qui font la force du XIX ${ }^{\mathrm{e}}$ siècle, celui justement émis par Jacques-Louis David de musées comme lieux d'éducation populaire. Un tel musée, à savoir un musée "d'antiques ", concourrait à former le goût des gens pour les "belles choses ", dans la lignée de l'appréciation de la statuaire antique comme idéal de beauté. Toutes les grandes collections étaient donc accessibles au public, à certains jours et horaires prédéfinis (souvent le dimanche), ou avaient volonté de l'être dès que les finances des universités permettaient le traitement d'un gardiennage ${ }^{23}$. La gratuité, de mise à l'époque dans tous les musées français, visait à faire venir en ces lieux les populations plus défavorisées ${ }^{24}$. Il est difficile d'appréhender aujourd'hui l'impact de ces collections dans la diffusion du goût pour l'antique, peu de sources témoignent de l'intérêt porté par les citoyens de l'époque à ces musées. Des cartes postales de vues de salles du musée universitaire de Lyon peuvent être considérées comme un gage de fréquentation (fig. 3), de même que l'épuisement rapide de la $3^{\mathrm{e}}$ édition de son catalogue. Des mentions éparses nous révèlent parfois le nombre important de visiteurs en ces lieux, le succès de certaines œuvres, le plan de Rome de Bigot à l'université de Paris

22 À l'exemple de Montpellier, comme en témoigne le plaidoyer de Ferdinand Castets, fondateur du musée des moulages, doyen de la faculté des lettres et conseiller municipal, Bulletin municipal du 31 janvier 1890. Note sur le Musée de Moulages de la faculté des lettres de Montpellier, Archives municipales de Montpellier, Registre des délibérations du conseil municipal, 1 D 74.

23 Un rapport d'André Joubin, chargé du cours d'archéologie à la faculté des lettres de Montpellier et responsable du musée des moulages, signale qu'en 1904 le musée de cette université n'était toujours pas accessible au grand public, faute de personnel de surveillance.

24 La suppression de la gratuité dans les musées français en 1922 ne semble pas concerner les musées universitaires. 
étant particulièrement prisé, de visites guidées, à Toulouse ${ }^{25}$ ou à Lille ${ }^{26}$. D'autres nous font prendre conscience que ces musées sont parfois inconnus de la population ${ }^{27}$.

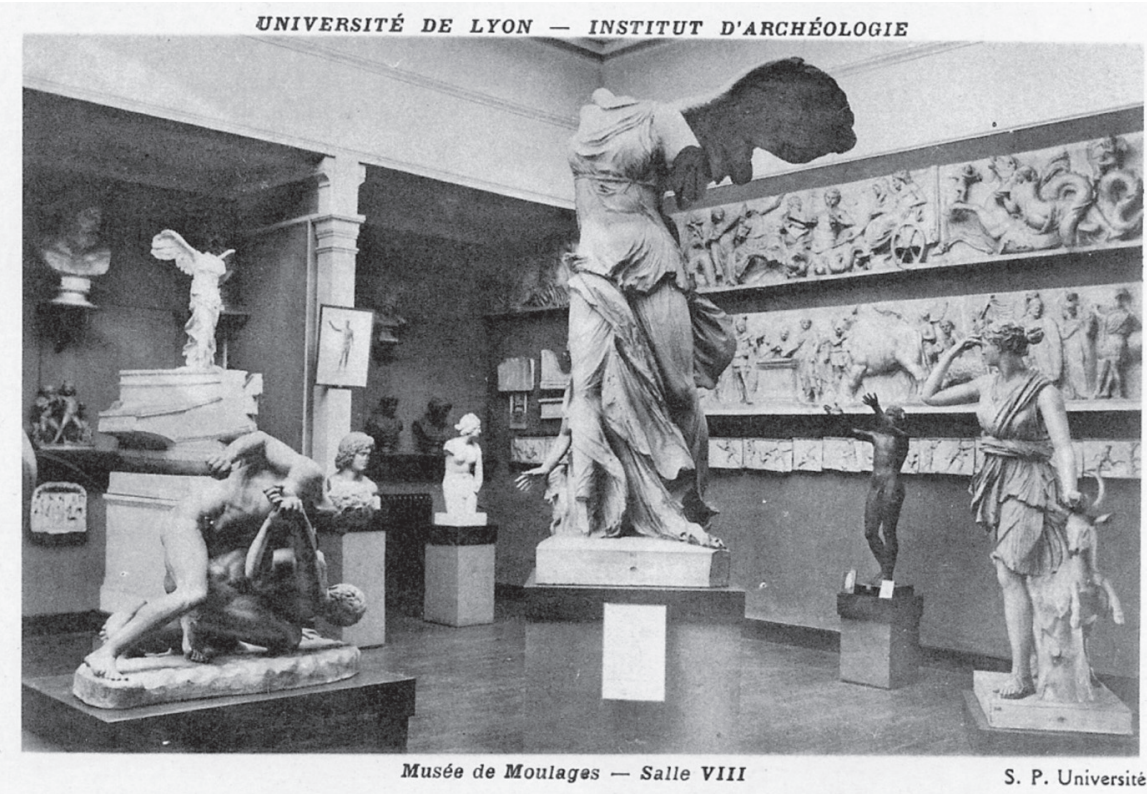

Figure 3. Carte postale du Musée des Moulages de la faculté des lettres de Lyon (C) Musée de Moulages - Université Lumière Lyon 2

Deux modes de visites étaient possibles, les visites libres ou guidées, l'un n'excluant pas l'autre au sein d'une seule université. Même si la simple vue des reproductions pouvait éveiller chez les gens une attirance pour ces arts, le musée se voulait un lieu d'éducation populaire et pour cela, avait été fait le choix d'une présentation non seulement esthétique, un arrangement des œuvres agréable à l'œil, mais aussi et surtout didactique, claire, ordonnée et explicite. Chaque œuvre était accompagnée d'un cartel, constituant une aide à la visite permettant d'identifier l'original moulé et donnant quelques informations essentielles mais succinctes (fig. 4 et 5). C'était le cas à Lyon, à Lille et à Montpellier après 1904. Dans d'autres universités, un simple numéro apposé

25 Elles sont relatées dans la revue L'Auta. Nouvelle série, Toulouse, n 55 (fév. 1923), nº 35 (fév. 1931), nº 55 (avril 1933), nº 218 (janv. 1952).

26 F. BENOÎT, op. cit., 1901, p. 538.

27 "N'ai-je pas entendu un maître de conférences d'une faculté voisine [...] me faire l'aveu qu'il n'avait jamais visité le Musée, qu'il n'en soupçonnait même pas l'existence ! ", Bulletin des amis de l'université de Lyon, Lyon, Impr. A. Storck et Cie, 1904. p. 45. Trois ans plus tôt, E. Morel avait plaidé pour que les ressources de l'Université fassent l'objet d'une publicité, pour une plus grande visibilité auprès des publics. 
sur le moulage ou le socle renvoyait au catalogue ou au guide du musée. Quand on sait qu'en 1905, à peine la moitié des musées de province possèdent un livret ${ }^{28}$, la publication de tels opus pour les musées universitaires révèle de manière significative la volonté pédagogique des professeurs d'archéologie. Rédigés par eux, parfois même par les étudiants comme à Bordeaux, ces ouvrages, que l'on considère comme des " catalogues populaires " davantage destinés aux visiteurs qu'aux étudiants, sont de véritables sources d'informations sur les œuvres, offrant au lecteur une courte analyse et des bibliographies sélectives mises à jour à chaque nouvelle édition. Ils étaient mis à la disposition du public, en vente ou en location auprès du gardien ou du concierge, et constituaient pour la visite un guide pratique, répertoriant salle par salle ou dans l'ordre chronologique de présentation les œuvres qui s'y trouvaient ${ }^{29}$. En outre, des photographies étaient souvent intégrées à la muséographie, restituant le contexte des œuvres originales, les édifices architecturaux qu'elles ornaient ou ornent encore, les essais de restitution ou l'indication des restaurations (fig. 3). Elles permettent de suppléer à la décontextualisation des œuvres et contribuent à la compréhension du discours muséal en fournissant des supports informatifs et visuels complémentaires.

28 Ch. Georgel, "Petite histoire des livrets de musées ", in Ch. Georgel (dir.), op. cit., 1994, p. 207-214.

29 Le premier catalogue de Montpellier (1890), celui de Bordeaux (1892) et de Toulouse (1895) présentent les œuvres dans une succession chronologique continue, tandis que le second de Montpellier (1904) et les trois catalogues de Lyon (1903, 1911 et 1923) adoptent une répartition par salles, sur le modèle des catalogues de la collection universitaire allemande de Strasbourg $(1887,1897)$. F. CASTETS, Catalogue du musée de moulages, Montpellier, C. Coulet, 1890, 64 p. ; P. PARIS (dir.), Université de France, faculté des lettres de Bordeaux, Musée archéologique, catalogue méthodique des moulages de sculpture grecque. Bordeaux : Impr. Ve Cadoret, 1889, 444 p. ; F. DürrbaCH, Musée de la faculté des lettres de Toulouse. Catalogue des moulages de la sculpture antique, Toulouse, Impr. A. Chauvin et Fils, 1895, 35 p. ; A. Joubin, université de Montpellier - Guide au musée de moulages de la faculté des lettres, Paris, Impr. Nationale, 1904, XI-67 p. ; H. LeCHAT, Annales de l'université de Lyon. Catalogues des collections universitaires, faculté des lettres, Catalogue sommaire du musée de moulages pour l'histoire de l'art antique, Lyon, A. Rey, 1903. XV-159 p. ; H. LeCHAT, Collection de moulages pour l'histoire de l'art antique, $2^{e}$ catalogue, Lyon, Impr. A. Rey \& C ie imprimeurs-éditeurs, 1911, XIII-232 p. ; H. LECHAT, université de Lyon, faculté des lettres, Institut d'histoire de l'art. Collection de moulages pour l'histoire de l'art antique, Lyon, Impr. A. Rey, 1923 (3 édition), XI-252 p. ; A. MichaELIS, Verzeichnis der Abguesse griechischer und ramischer Bildwerke im kunstarchaeologischen Institut der KaiserWilhelms-Universitaet Strassburg, Strasbourg, Verlag von Karl J. Trübner, 1887, 69 p. ; A. Michaelis, Führer durch das archäologische Museum der Kaiser-Wilhelms-Universität Strassburg, Strasbourg, Verlag von Karl J. Trübner, 1897, 137 p. 


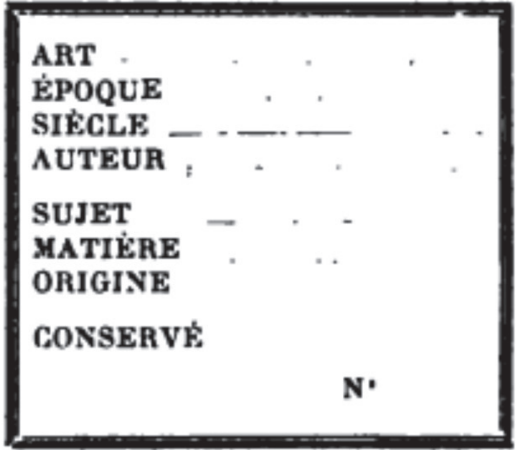

Figure 4. Type de cartel proposé par François Benoît (Lille)

(C) F. BENOIT, Revue internationale de l'enseignement, vol. 41, 1901, p. 537

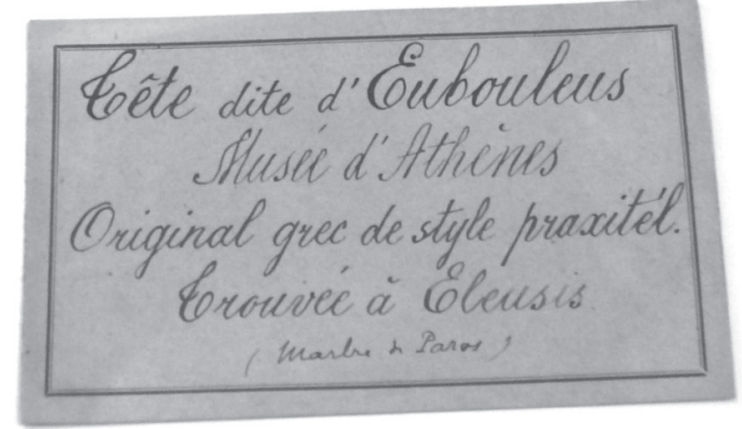

Figure 5. Cartel manuscrit provenant du musée des moulages de Montpellier

(C) S. Morinière

Pourtant, si les visiteurs étrangers ont contribué à la renommée de ces collections, qui devenaient alors la «vitrine » de l'Université ${ }^{30}$, un « joyau » montré lors des visites officielles ${ }^{31}$, le principal public extérieur visé restait, dans les pensées des professeurs, les populations locales. Une conviction qui n'est pas sans rapport avec la volonté du ministère de l'Instruction publique d'ancrer plus profondément les universités dans leur région ${ }^{32}$. Ferdinand Castets (1838-1911 ?), doyen de la faculté des lettres de Montpellier et fondateur du musée des moulages de cette université, espérait faire de cette collection un moyen de sensibiliser la population au patrimoine archéologique de la région. Dans le Compte rendu des travaux des facultés pour l'année scolaire 18881889, il écrivait ces mots : "Supposez l'existence dans notre région d'un enseignement d'archéologie artistique, et l'on sera moins disposé à laisser partir nos trésors pour des musées lointains ${ }^{33}$." Car le problème est là, l'inculture des populations locales conduit

30 Visite du musée des moulages de l'université lyonnaise à Lord Glenesk et une délégation d'Écossais en 1904, Bulletin de la Société des amis de l'université de Lyon, op. cit., 1904. p. 45.

31 Visite du président de la République M. Fallières, du ministre de l'Agriculture M. Ruau, du ministre du Commerce M. Doumergue, du directeur de l'enseignement supérieur M. Bayet, du maire de Lyon M. Herriot, et d'autres personnalités politiques éminentes le 20 mai 1907, Bulletin de la Société des amis de l'université de Lyon, Lyon, Impr. A. Storck et Cie, 1906-1907, p. 113-120.

32 Orientation universitaire initiée par Louis Liard, directeur de l'enseignement supérieur de 1884 à 1902.

33 Il pense en disant cela à la copie romaine du Diadumène découverte à Vaison-la-Romaine et acquise par le British Museum et à la Vénus d'Arles, offerte à Louis XIV et conservée ensuite au musée du Louvre. F. CASTETS, Rapport sur les travaux de la faculté des lettres 
à de tristes séparations. La réplique romaine du Diadumène de Polyclète découverte en 1862 à Vaison-la-Romaine par l'agriculteur M. Jacquet, est vendue à Eugène Raspail de Gigondas qui le cède en 1869 au British Museum de Londres suite au refus du musée du Louvre et du musée Calvet d'Avignon de l'acquérir. La même année, la collection Véran Blanchon rejoint le musée des antiquités nationales de Saint-Germaine-en-Laye. Malgré quelques actions locales dans cette région, notamment l'abbé Joannis qui abrite une partie des découvertes archéologiques vaisonnaises dans le cloître, les collectionneurs préferent vendre des œuvres à de grands musées plutôt qu'en faire don à la municipalité qui manque de crédits pour l'acquisition et la conservation des œuvres ${ }^{34}$. Les richesses de l'antique ville de Vasio se retrouvent ainsi dispersées partout en France et à l'étranger. Les musées de moulages seraient donc vus comme une solution «moralisatrice » mettant en exergue le patrimoine local. C'est pourquoi, à côté des poncifs de la statuaire grecque, se trouvent ponctuellement des œuvres issues des fouilles régionales. À Toulouse ce sont des découvertes provenant du site de Martres-Tolosanes, parmi lesquelles les têtes d'une Aphrodite et d'Auguste couronné, toutes deux conservées aujourd'hui au musée Saint-Raymond. À Montpellier ce sont plutôt des sculptures du midi de la France, tels la Vénus d'Arles (fig. 6, à gauche), les Aphrodite de Fréjus et de Vienne, le Diadumène de Vaison-la-Romaine ou encore une tête de Platon d'Aix-en-Provence. À Lyon sont exposées quelques œuvres conservées dans les musées municipaux de la ville, la statue féminine archäque dite "Corè de Lyon ", la Junon découverte à Vilette-Serpaize, près de Vienne, la tête d'Hercule de Thizy, le bustehermès de Zénon de Kitios trouvé à Lyon ou encore la statuette de Zeus provenant de Lyon mais propriété du British Museum.

À travers l'étude des gypsothèques universitaires, c'est le climat politique, social et intellectuel de la fin du XIX $x^{\mathrm{e}}$ siècle et du début du siècle suivant qui prend forme. Ces collections sont des témoignages de l'engouement du XIX siècle pour la constitution de musées et l'appropriation des moulages comme objets muséaux au même titre que les œuvres originales. Elles révèlent les liens importants entre les musées et l'enseignement, entre l'art et l'État ${ }^{35}$. Par leur ouverture à des publics différents, par leur organisation scientifique et didactique, et par leur composition, ces musées universitaires deviennent des moyens de diffusion d'une Antiquité modèle. Révélateurs du scientisme de la Troisième République, vecteurs d'une identité patrimoniale plus ou moins affirmée, ils ont offert aux visiteurs un aperçu de l'art grec et romain, une vision " complète " parfois mise en regard avec d'autres périodes, à l'instar de la Renaissance considérée

de Montpellier pendant l'année scolaire 1888-1889, Montpellier, impr. Jean Martel Ainé, 1889 , p. 8.

34 Ch. BEZIN, "L'archéologie vaisonnaise ». Site internet du musée de Vaison-la-Romaine http://www.vaison-musees.com/archeologie.php

35 Ces relations ne sont pas spécifiques aux musées universitaires, mais découlent également de l'histoire des autres musées français. D. Poulot, Musée et muséologie, Paris, éditions La Découverte, 2009 (nouvelle édition), p. 68-69. 
comme le renouveau du goût pour l'art antique, et complétées par des moulages des époques principales de l'art (médiévale, moderne, contemporaine) sans toutefois aller jusqu’à la muséologie comparative qui a régi les débuts du musée parisien de Sculpture comparée.

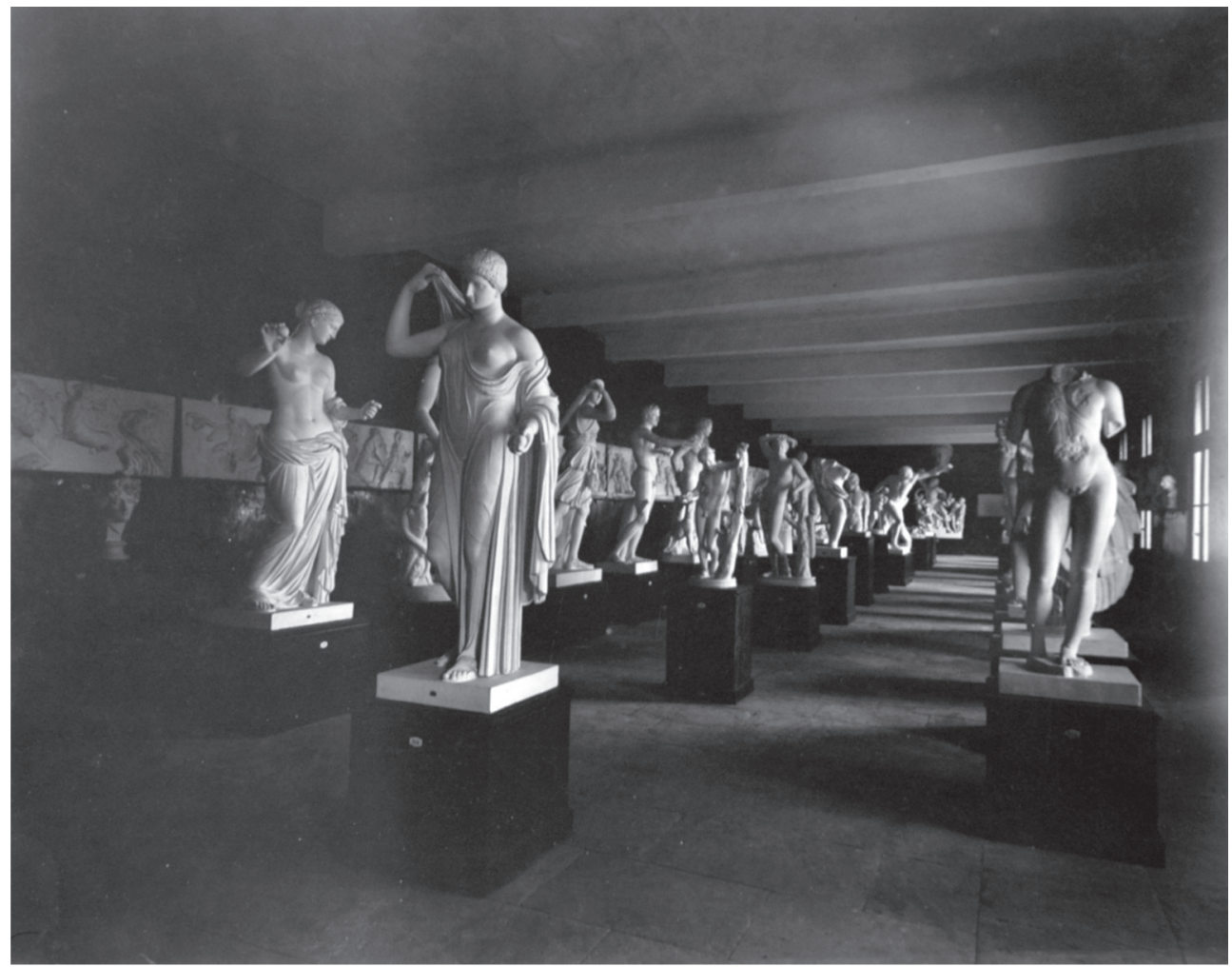

Figure 6. Musée des Moulages de la faculté des lettres de Montpellier. ca 1890-1904. (C) Musée des Moulages de l'Université Paul-Valéry Montpellier 3.

Soline MORINIÈRE

Doctorante en histoire de l'art contemporain à l'Université de Bordeaux

Chargée d'études documentaires

Service régional de l'Archéologie

DRAC Alsace

soline.moriniere@ulture.gouv.fr 